\title{
44 BIKER'S SCAPULA: AN ERGONOMIC IMPLICATION OF
} BIKING

Shantanu Sharma, ${ }^{1}$ Ram Prabhu, ${ }^{2}$ Mrigank Aggarwal ${ }^{3}$ 'Manav Rachna International University, Faridabad, Harayana, India; ${ }^{2}$ Pt. Deendyal Upadhyaya Institute for the

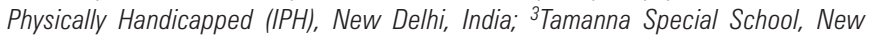
Delhi, India

10.1136/bjsm.2010.078725.44

Introduction There are emerging trends of designing motor bikes with sporty looks. These bikes are especially popular among youth due to the speed that it can gain. There are bikers who complain of pain after biking for long distances. This is generally a case with heavy, non-cruiser bikes. The purpose of this poster is to depict the biomechanical and ergonomical basis of this pain felt at the superior angle of the scapula along with the diagnosis and treatment. Description: (1) Pain: the pain (dull and aching type) occurs at the superior angle of the scapula. After driving a bike, the pain stops but the soreness remains for 4-5 days. Any activity involving shrugging brought on the pain. (2) Biomechanics and Ergonomics: to streamline the body according to the speed of the bike, the biker pokes his chin, shrugs his shoulder and flexes his elbow. In this position, levator scapulae muscle is been used to shrug the shoulder as well as to maintain the position of the scapula. As this position is dynamic, the position keeps the levator scapulae in a contracted phase for prolonged period which may lead to repetitive stresses and microtrauma at the insertion of levator scapulae and start causing pain. (3) Diagnosis: eight patients were identified and this type of pathology is suspected wherein superior angle of scapula is tender and resisted shoulder shrugging is painful at the same area. However, scapular mobility and arm movements are normal. (4) Treatment: ultrasound therapy is definitely effective in relieving symptoms in two to three sittings after which resisted shrugging is given with mild resistance. However, it was seen that the pain recurs after a week or so if the long distance biking continues. 
(5) Prevention: To avoid this, bikers must take care of their posture while riding as well as proper selection of bike. It is also advised to use cruiser bikes for long distances.

Conclusion In future, the cases with the similar history must be properly assessed and documented so that the further research can be carried out on the causes, permanent treatment and the other ways to prevent it. 